\title{
Low threshold unmyelinated mechanoafferents can modulate pain
}

\author{
Kathrin Habig ${ }^{1^{*}}$ D, Anne Schänzer², Wolfgang Schirner ${ }^{1}$, Gothje Lautenschläger ${ }^{1}$, Benjamin Dassinger ${ }^{3}$, \\ Håkan Olausson ${ }^{4}$, Frank Birklein ${ }^{5}$, Elke R. Gizewski ${ }^{6}$ and Heidrun H. Krämer ${ }^{1}$
}

\begin{abstract}
Background: Human, hairy skin contains a subgroup of C-fibers, the C-low threshold mechanoreceptive afferents ((C-LTMR) C-tactile or C-touch (CT) fibers) that are linked with the signaling of affective aspects of human touch. Recent studies suggest an involvement of these afferents in the modulation of pain in healthy volunteers. Small fiber neuropathy (SFN) is associated with a damage of C-fibers. Therefore, an impairment of C-LTMRs can be assumed. We aimed to elaborate a possible role of CT-afferents in pain modulation by investigating healthy volunteers and SFN-patients.
\end{abstract}

Methods: Experiment I: 20 SFN-patients (12 women, median age 52.0 years) and 20 healthy controls (14 women, median age 43.0 years) participated in this prospective fMRI and psychophysical study. Heat-pain (HP), CT-targeted touch (slow brushing) and HP combined with CT-targeted touch were applied in randomized order to the left shank in a block design. The participants rated pain intensity on a visual analogue scale. Experiment II: We investigated a possible impact of pain intensity on $C T$ induced pain modulation (10 healthy participants). The intensity of HP stimulation was chosen to induce pain intensity 50/100 (NRS). HP stimulation was applied with and without CT-targeted touch.

Results: Experiment I: $C$-stimulation was sufficient to reduce heat pain in healthy participants $(p=0.016)$, but not in SFN-patients. HP induced pain intensity was significantly higher $(32,2$ vs 52,6$)$ in SFN-patients. During HP, bold responses in pain associated areas were observed in both groups. Additional CT-stimulation elicited no significant difference of bold responses compared to HP. Experiment II: In healthy volunteers, we reproduced a significant reduction of HP intensity by CT-stimulation $(p=0.038)$.

Conclusions: $C T$ input seems to be sufficient to modulate pain, independent of intensity of the pain stimulus. As a prerequisite, the $C T$ fibers have to be intact as in healthy volunteers. If $C T$ fibers are impaired - as in SFN -, CT-targeted touch does not modulate pain intensity. The location of CT-induced pain modulation might be attributed to the level of the dorsal horn since the cortical activation pattern of heat pain with and without CT-targeted touch did not differ in healthy subjects and in SFN-patients.

Keywords: $\subset$ afferents, Small fiber neuropathy (SFN), Pain perception, Pain inhibition, Spinal cord, fMRI

\section{Background}

In human hairy skin, pleasant touch is signaled by $A \beta$ afferents and a subgroup of $\mathrm{C}$ fibers, the $\mathrm{C}$-low threshold mechanoreceptive (C-LTMR; CT $=\mathrm{C}$ touch or $\mathrm{C}$ tactile) afferents. Those fibers were first detected using microneurography of the infraorbital nerve [1], but were then found to be distributed more widely in the arm and leg [2]. They do not appear in glabrous skin [2, 3].

\footnotetext{
* Correspondence: kathrin.habig@neuro.med.uni-giessen.de

${ }^{1}$ Department of Neurology, Justus Liebig University, Klinikstr. 33, 35392

Giessen, Germany

Full list of author information is available at the end of the article
}

Characterization of C-LTMR revealed that these afferents react to low mechanical indentation forces $(<5 \mathrm{mN})$ [4] and to slowly moving stimuli $[5,6]$ as in gentle stroking. The pleasantness of touch is positively correlated with the firing rate of these fibers [6]. In mice, the cell bodies of the C-LTMRs are randomly distributed in the dorsal root ganglia, C-LTMRs terminate in Lamina II of the dorsal horn accompanied by $A \delta$ and $A \beta$ afferents [7]. They enter the lamina I/II spinothalamic pathway up to the ventromedial posterior thalamic nucleus $[8,9]$. 
In humans, the main brain areas receiving C-LTMR information belong to the somatosensory system and affect processing brain networks like the contralateral posterior insular cortex [10] or the medial prefrontal cortex [11]. The intensity of CT targeted touch is encoded in the primary and secondary somatosensory cortex (S1 contralateral, S2 bilateral), whereas the pleasantness is encoded in the pregenual anterior cingulate cortex [12]. C-LTMRs also activate regions involved in reward processing (putamen and orbitofrontal cortex $[13,14]$ ) and in processing of social stimuli (posterior superior temporal sulcus [15-17]).

Animal experiments indicate that $\mathrm{CT}$ activation reduces pain, but the precise anatomical localization of this phenomenon is still unknown [18-20]. In humans, slow and gentle touch is supposed to ease pain [21]. Recently a modulation of laser evoked pain by CT targeted touch on the contralateral extremity has been reported. The effect of CT targeted touch (attenuating/ increasing pain) was dependent on the attachment styles (attachment anxiety/ attachment avoidance) of the participants [22]. It needs to be further elucidated, whether an inhibition of nociceptive neurons by C-LTMRs exists in humans and additionally, where this is being processed. An ideal model to answer this question is the investigation of small fiber neuropathy (SFN) patients. SFN is defined as a neuropathy that exclusively affects the thin sensory ( $\mathrm{C}$ and $\mathrm{A} \delta$ fibers) nerve fibers [23]. It is confirmed by thermal testing and skin biopsy. Although C-LTMRS cannot be easily tested, it can be assumed, that C-LTMRs as a subgroup of $\mathrm{C}$ fibers should be impaired as well.

In the present study, we examined healthy volunteers with intact C-LTMRs and SFN patients with supposedly impaired C-LTMRs to investigate the impact of C-LTMR signaling on pain modulation in humans. We compared heat pain intensity with and without CT targeted touch (gentle stroking with a soft brush) in both groups. CT targeted touch was proposed to attenuate pain in healthy volunteers and to a lesser extend in SFN patients. To search for possible CNS mechanisms, we performed fMRI.

\section{Methods}

\section{Participants}

Twenty patients with SFN (12 women, 8 men, median age: 52 years, age range: $35-71$ years) and 20 healthy controls (14 women, 6 men, median age: 44 years, age range: $30-75$ years) were included in the study. All participants were fully right handed according to a modified handedness score [24].

In all SFN patients, the medical history was recorded and a thorough neurological examination was performed. SFN was diagnosed according to the diagnostic criteria by Hoeijmakers et al. [25]. In brief, all patients suffered from length dependent neuropathic pain in the feet and lower legs. In all patients, cold (CDT) and warm detection thresholds (WDT) were determined using quantitative sensory testing (QST). Every SFN patient underwent a skin biopsy. The intraepidermal nerve fiber density (IENFD) in skin punch biopsies was analyzed according to the recommended guidelines to confirm the diagnosis of SFN [26, 27]. Electroneurography was performed to exclude large fiber involvement. SFN was diagnosed if the patients presented with typical clinical features [25], pathological thermal detection thresholds as well as decreased IENFD. Patients were excluded from the study if they showed abnormalities in the clinical neurological examination especially in vibration detection thresholds or pathological values in the motor and sensory nerve conduction studies. Patients that presented with allodynia were also excluded from the study since CT targeted touch would elicit pain in these patients [28].

In healthy volunteers, the medical history, the clinical neurological examination as well as the thermal testing had to be unremarkable.

All participants gave their informed written consent according to the latest revision of the Declaration of Helsinki. The study was approved by the local ethics committee of the Justus-Liebig-University, Gießen (105/11).

\section{Skin biopsy}

In SFN patients, skin punch biopsies $(3 \mathrm{~mm})$ were taken $10 \mathrm{~cm}$ above the malleolus lateralis in the innervation territory of the sural nerve and the tissue samples were analysed according to the recommended guidelines [26, 29]. In brief the tissue samples were fixed in $2 \%$ Zamboni solution and cut into $50 \mu \mathrm{m}$ thick slices. Immunofluorescence staining was performed at free floating sections with antibody against PGP 9.5 (rabbit polyclonal anti-PGP 9.5 1:1000; zytomed) and a second antibody (goat anti-rabbit Alexa Fluor 488, 1:1000; invitrogen). In a minimum of six sections the intraepidermal nerve fibers (IENF) were analysed using a Leica Immunofluorescence Microscope DM 2000 at magnification 400 fold. IENFD was calculated as the mean of IENF per millimetre.

\section{Cold and warm detection thresholds}

All participants were tested for cold and warm detection thresholds on the dorsum of the left foot. The exact procedure has been already described elsewhere [30]. In brief, warm and cold detection thresholds were determined using a TSA 2001-II (MEDOC, Israel; contact area of the thermode: $9 \mathrm{~cm}^{2}$; baseline temperature: $32{ }^{\circ} \mathrm{C}$; slope: $1{ }^{\circ} \mathrm{C} / \mathrm{s}$ ). The mean threshold temperature of 3 consecutive measurements was calculated.

\section{Experiment I: stimulation protocol}

During fMRI, three different conditions were applied at the left lower leg in a randomized order: 1. heat pain 
only, 2. heat pain and CT-targeted brushing and 3. CT targeted touch only. The heat pain elicited pain was rated on a computerized rating scale that was presented via a projector. Each condition was applied for $10 \mathrm{~s}$ alternating with rest for a total of 6 times each. The heat pain- and tactile stimulus were synchronized to the MRimages via an electrical trigger from the scanner. The trigger has been linked to a matlab-based program showing a time scale, which has been presented on a screen in the scanner room. The screen could be seen inside (CT stimulation) and outside (heat pain stimulation) of the scanner room. The timescale started with the sequence "rest" and a countdown signalizing stimulus onset, which has been additionally marked as a color change, so that the investigators were primed for the final optical trigger to start the stimulation manually. The rating period started $5 \mathrm{~s}$ after stimulus onset and was presented on the same screen. See Fig. 1. When an error of timing occurred, the sequence has been excluded.

We applied all conditions in randomized order to minimize order effects, all participants were blinded for the expected pain inhibition by CT stimulation. Participants were unaware of the aims of the study. They were only informed that central activation patterns and pain intensity during heat pain and touch are investigated in various combinations.

\section{Heat pain stimulation}

Heat pain thresholds (HPT) were determined in all participants at the left foot within the L5 dermatome. The mean HPT was calculated from 3 consecutive measurements according to the method of limits (for detail see: [31]. The individual heat pain threshold was determined directly before the subject entered the scanner, to obtain stable heat pain intensities the applied temperature was $\mathrm{HPT}+1.5^{\circ} \mathrm{C}$. The intended pain intensity was characterized as "painful but bearable". Heat pain stimulation was carried out at the participants left foot.

\section{Tactile stimulation}

Repetitive strokes with a soft painter's brush $(2 \mathrm{~cm}$ wide, stimulation force $0.8 \mathrm{~N}$; stimulation velocity $3 \mathrm{~cm} / \mathrm{s}$ ) were applied in proximal to distal direction on the left lower leg (CT targeted touch). The stroking was performed over a length of $30 \mathrm{~cm}$ with a velocity of $3 \mathrm{~cm} / \mathrm{s}$ on the lateral lower leg in the area of hairy skin in the dermatome L5. To avoid inter-investigator variability stroking was always performed by the same investigator $(\mathrm{KH})$.

\section{Pain rating}

Subjects were asked to quantify the heat derived pain intensity on a numeric rating scale (NRS; $0-100$ ) with the anchors 0 "no pain" on the left end and 100 "worst pain imaginable" on the right end. The rating started $5 \mathrm{~s}$

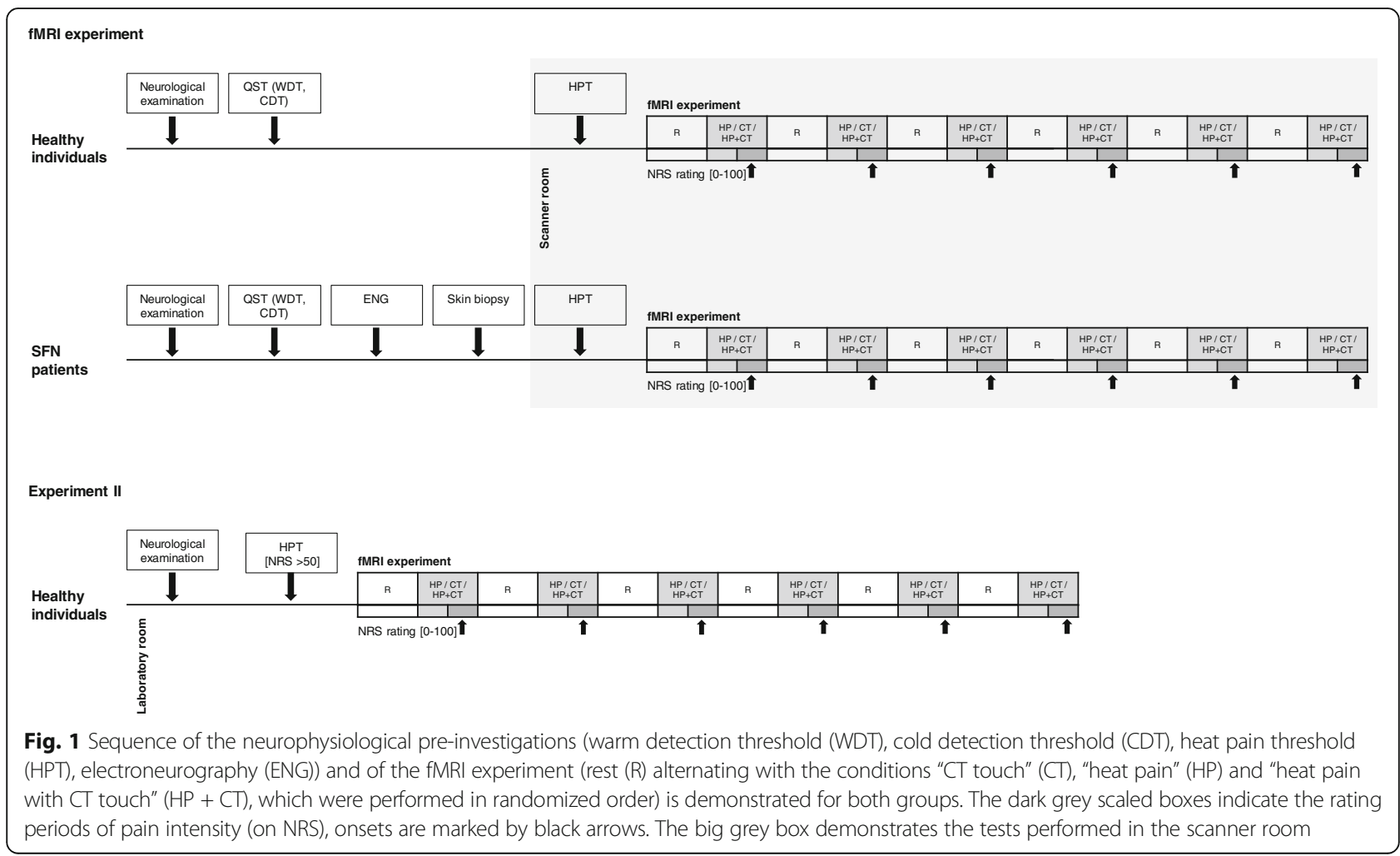


after the beginning of each trial and lasted for $5 \mathrm{~s}$. The computerized NRS was presented via a projector. Pain ratings were assessed by operating a fiber optic response pad (Current Designs, Inc., Philadelphia, USA) which was connected to a computer. The pad was held in the dominant right hand by all subjects. The handling of the NRS was practiced before the scanning. See Fig. 1.

\section{fMRI}

\section{Data acquisition}

Functional and anatomical MR scans were performed in a 3 Tesla MR Scanner (Siemens Magnetom Verio, Siemens Medical Systems, Erlangen, Germany) using the 8 channel standard head coil. For structural images, a T1 weighted sequence (TR $1900 \mathrm{~ms}$, TE $2.45 \mathrm{~ms}$, bandwidth $170 \mathrm{~Hz} /$ Pixel, TI $900 \mathrm{~ms}$, flip angle $9^{\circ}$, FOV $176 \times 512 \times 512 \mathrm{~mm}$, orientation: sagittal, voxel size $1 \times 1 \times 1 \mathrm{~mm}$ ) was acquired. Functional images consisted of EPI sequences (TR $2800 \mathrm{~ms}$, TE $30 \mathrm{~ms}$, bandwidth $2232 \mathrm{~Hz} /$ Pixel, flip angle $90^{\circ}$, FOV $240 \times 240 \times 120 \mathrm{~mm}$, orientation: $\mathrm{T}>\mathrm{C}-21.4,40$ slices, voxel size $3 \times 3 \times 3 \mathrm{~mm}^{3}$, TA 5:43).

\section{Data processing}

For data processing, we used Statistical Parametric Mapping (SPM8, Welcome Department of Cognitive Neurology, London, UK). Prior to first level analysis, the images underwent preprocessing. This included realignment to the mean functional image (b-spline interpolation), segmentation of the structural image and normalization into Montreal Neurological Institute (MNI) space. Data were smoothed with full-width and half maximum (FWHM) of $6 \mathrm{~mm}$ of the Gaussian smoothing kernel.

A first level analysis was performed for each subject individually using the general linear model with a highpass filter with a cutoff of $128 \mathrm{~s}$. Contrasts were defined for the different stimuli (heat pain and heat pain combined with CT-targeted touch) vs rest. Subsequently mixed-effects group analyses were performed for healthy subjects and SFN patients separately with a one sample t-test. Additionally, two sample t-tests were used for the evaluation of group differences for the conditions "heat pain" versus "heat pain and CT targeted touch" between healthy participants and SFN patients.

Supplementary, we performed a region of interest (ROI) analyses using the small volume correction feature of SPM. As ROIs we chose the projection sides of CT fibers (insula, medial prefrontal cortex (MPFC) and pregenual ACC). The mask for the insulas were derived from the wfu pickatlas (human atlas, TD labels) for insula bilaterally. For masking the MPFC and pregenual ACC spheres with a diameter of $10 \mathrm{~mm}$ were placed according to Liljencrantz and Björnsdotter (medial frontal gyrus, MNI coordinates $11,52,-6$; $-11,52,-6$; [11]) and Case
2016 (pregenual ACC, MNI coordinates - 8, 50, 6; 8, 50, 6 ; [12]). These regions were saved as one image and used as inclusive mask for an additional ROI analysis.

Analysis was performed on a voxel-level and corrected for multiple testing by random field theory with a family-wise error rate of $\alpha=0.05$.

For anatomical labelling, FSL based atlases were used (Harvard-Oxford Cortical Structural Atlas; HarvardOxford Subcortical Structural Atlas; Juelich Histological Atlas (http://fsl.fmrib.ox.ac.uk/fsl/fslwiki/Atlases)).

For visualization of the results, we used a standard template (SPM 8 template avg152T2), only showing significant activations.

\section{Experiment II}

In the second experiment, we investigated 10 healthy individuals in our laboratory rooms. "Heat pain" and "heat pain with CT targeted touch" were applied to the left foot (within the L5 dermatome), CT stimulation was performed on the left shank by slow brushing $(3 \mathrm{~cm} / \mathrm{s})$, according to the setup in our first experiment during fMRI. The stimulations were applied in a randomized order lasting $10 \mathrm{~s}$ each with $10 \mathrm{~s}$ of rest in between stimulations. Heat pain stimulation was chosen to elicit pain intensities $>50$ on a NRS (0-100; anchors 0 "no pain" and 100 "worst pain imaginable"). The rating started $5 \mathrm{~s}$ after the beginning of each trial and lasted for $5 \mathrm{~s}$. NRS rating was performed with the same computerized NRS which we used during the experiment in the scanner room, but was presented on a laptop. Pain ratings were delivered by pressing a computer key.

\section{Statistics}

Psychophysical data were analyzed using the SPSS Statistics (IBM, Version 23.0 for Windows) software package. Kolmogorov-Smirnov tests of normality were run for all data sets and parametric or non-parametric statistics were used accordingly as described in the experimentspecific results. All values are given as medians and interquartile range (IQR) in the case of a non-normal distribution and as means \pm standard deviation (SD) in the case of a normal distribution. Values were considered significant if $\mathrm{p}<0.05$.

\section{Results}

\section{Skin biopsies}

The mean intraepidermal nerve fiber density (IENFD) in all SFN patients was decreased compared to normal control values (mean IENFD: 2.46 (SD 1.65); norm: 4.0 to 7.8 [27]).

\section{Cold and warm detection thresholds (CDT and WDT)}

SFN patients had significantly higher warm detection thresholds (SFN patients: median $43{ }^{\circ} \mathrm{C}$, IQR 5.3; healthy 
participants: $39.8{ }^{\circ} \mathrm{C}$, IQR 6.3; p <0.001) and lower cold detection threshold (SFN patients: median $24.1{ }^{\circ} \mathrm{C}$, IQR 5.7; healthy controls: median $29.0{ }^{\circ} \mathrm{C}$, IQR 2.9; $p=0.003)$. These findings support the impairment of $\mathrm{A} \delta$ - (CDT) and C-fibers (WDT) in accordance with diagnosis of SFN.

\section{Heat pain thresholds (HPT)}

SFN patients had significantly higher heat pain thresholds compared to the controls (HPT healthy participants: median $46{ }^{\circ} \mathrm{C}$, IQR 3; HPT SFN patients: median $47^{\circ} \mathrm{C}$, IQR $\left.1 ; p=0.045\right)$.

\section{Pain ratings during $\mathrm{fMRI}$}

In healthy subjects, heat pain intensity was significantly reduced by the addition of CT targeted touch (NRS heat pain only: mean 32.2 , SD 22.35; NRS heat-pain and CT targeted touch: 28.6, SD 21.86; $p=0.016$, paired t-test). For details see Fig. 2.

In SFN patients, heat pain intensity was not altered by the addition of CT targeted touch (NRS heat pain only: mean 52.6, SD 20.2; NRS heat-pain and CT targeted touch: 57.2, SD 23.08; $p=0.139$, paired t-test). For details see Fig. 2.

Pain ratings for the conditions heat pain $(p=0.005)$ and heat pain with $\mathrm{CT}$ stimulation $(\mathrm{p}<0.001)$ were significantly higher in SFN patients compared to controls. For details see Fig. 2.

\section{fMRI}

\section{Bold responses for "CT targeted touch" only}

CT targeted touch in healthy subjects showed bold responses in the left postcentral gyrus and the right

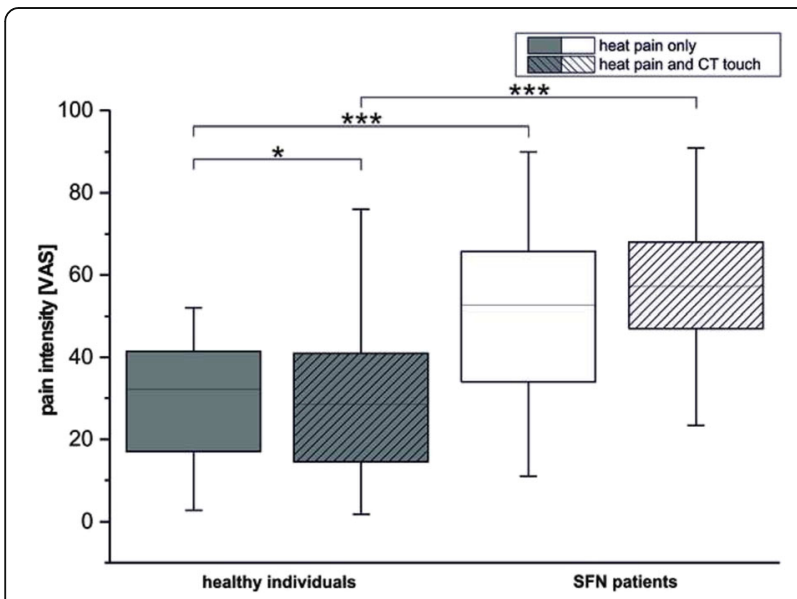

Fig. 2 Visual analogue scale (NRS) ratings for the conditions "heat pain only" and "heat pain combined with CT stimulation" in healthy subjects and SFN patients. $C T$ stimulation significantly reduced of heat pain intensity in healthy subjects $(p=0.016)$ but not in SFN patients $\left({ }^{*} p \leq 0.05\right.$, ${ }^{* *}$ of $p \leq 0.01,{ }^{* * *}$ of $p \leq 0.005$ ). The overall rating of pain intensity for both conditions was significantly higher in the SFN patients (heat pain only: $p=0.005$; heat pain combined with $C T$ targeted touch: $p<0.001$ ) parietal operculum (supramarginal gyrus). In SFN patients, CT-targeted touch resulted in activation of the right parietal operculum (supramarginal gyrus). For details see Fig. 3 and Table 1.

No significant difference of bold responses could be detected between healthy controls and SFN patients in the condition "CT targeted touch" only.

\section{Bold responses for "heat pain" only}

In healthy subjects, the heat pain stimulus evoked activations of areas known to be associated with pain processing: anterior insular cortices bilaterally (right 33,23,1; T 10.0; left - 30,23,1; T 10.1) and the anterior cingulate cortex (cluster peak 9,20,31; T 7.2). For details see Fig. 3 and Table 1. In SFN patients the condition "heat pain" also evoked activations in cortical areas associated with pain processing (e.g. anterior cingulate cortex bilaterally (cluster peak at 3,11,46; $\mathrm{T}$ 8.7), the insular cortex bilaterally (right anterior-middle insular cortex: 42,17,-2; T 7.2 and left anterior insular cortex:27,20,-5; T 8.3). For details see Fig. 3 and Table 1.

No differences in the cortical activation could be detected between healthy controls and SFN patients for the condition "heat pain" only.

\section{Bold responses for "heat pain and CT touch"}

In healthy volunteers, heat pain and CT targeted stimulation evoked significant bold responses in the anterior insula bilaterally, the ACC and the supramarginal gyrus. Right hemispherical activations correspond to the putamen, frontal opercular cortex, middle frontal gyrus, anterior intra-parietal sulcus, inferior parietal lobule, inferior frontal gyrus and activation of less specified brain regions. There were no supplementary bold responses in the left hemisphere. For details see Fig. 3 and Table 1.

In SFN patients, heat with CT touch elicited bilateral bold responses of the insula, ACC, supramarginal gyrus, the opercular cortex and the anterior intra-parietal sulcus. In addition, the right paracingulate gyrus showed a significant bold response. For details see Fig. 3 and Table 1.

There was no difference of bold responses comparing the conditions "heat pain only" and "heat pain with CT targeted touch" in both groups.

A sensitive ROI analysis for all performed tests detected no additional bold responses in the predefined areas (insula, medial frontal gyrus and pregenual ACC).

\section{Experiment II}

Since heat induced pain intensity was higher in SFN patients compared to our controls, we performed an additional psychophysical experiment (experiment II) in healthy subjects to investigate a possible impact of pain intensity on CT mediated pain modulation. The heat stimuli were set to induce pain intensity NRS 50 from 


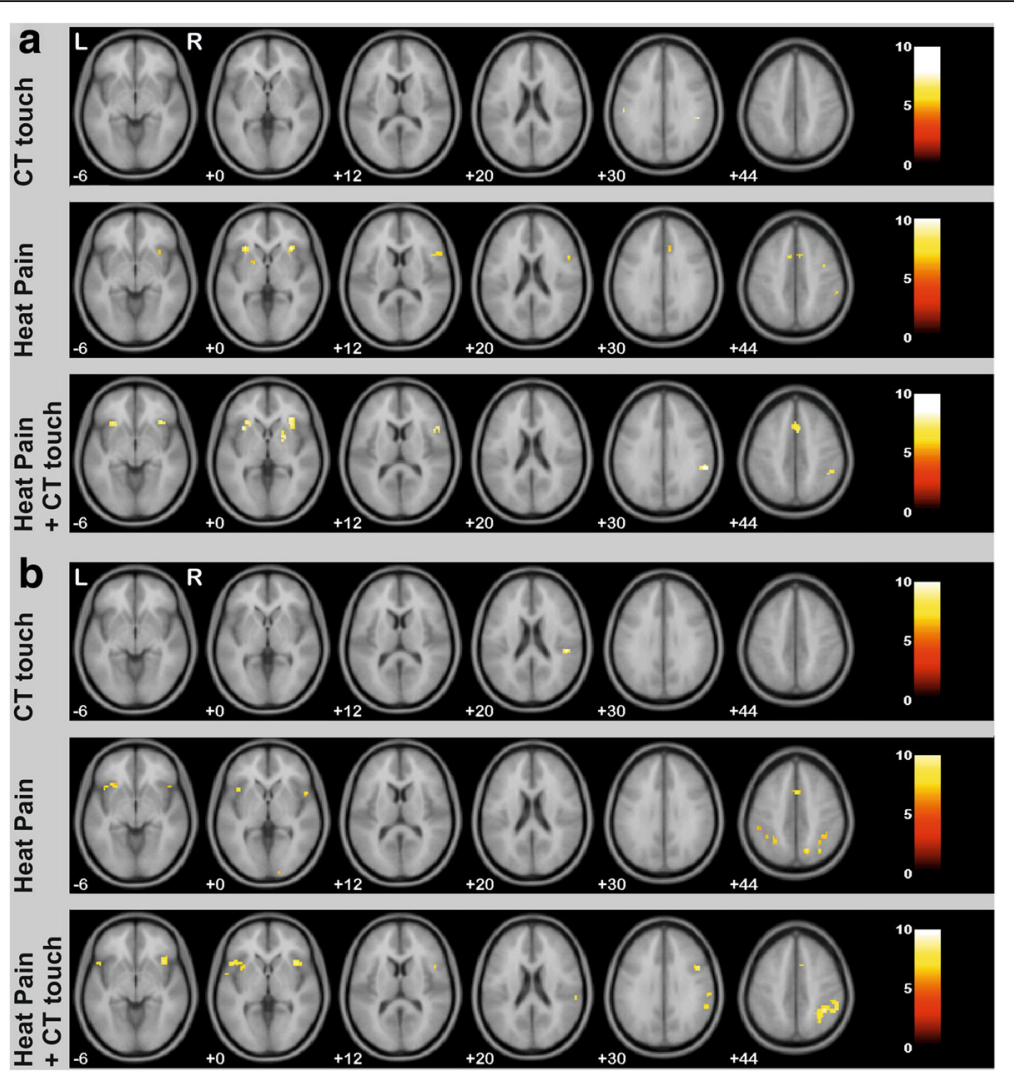

Fig. 3 fMRI images for a healthy subjects and $\mathbf{b}$ SFN patients, showing neuronal activation for the main effect of " $\mathrm{CT}$ touch", "heat pain only" and "heat pain combined with CT targeted touch". The right side of the images corresponds to the right hemisphere. Bold responses are shown with FWE correction, $p<0.05$ and cluster size $>3$ voxel. Results are shown on SPM template avg152T2

100 (applied temperature median $47.0{ }^{\circ} \mathrm{C}$, IQR 3.25). With this setup, we reproduced the significant reduction of heat pain by CT stimulation despite high pain intensities (NRS heat pain only: mean 55.9, SD 15.26; NRS heat-pain and CT targeted touch: 48.4, SD 16.97; $p=0.038$, paired t-test).

\section{Discussion}

We provide psychophysical evidence that C-LTMR activation modulates experimental heat pain in humans. This notion is largely independent of the stimulus induced pain intensity. In SFN, in which CT fibers are presumed to be impaired, CT activation does not alter heat pain. Since the thick myelinated $A \beta$ fibers were intact in both groups, these afferents were assumed to be less involved in pain reduction. The mechanisms how CT modulated pain relief is being processed remain unclear. We did not detect significant differences in cortical activation patterns between heat stimulation with and without CT targeted touch.

\section{Pain modulation}

Heat pain intensity was significantly reduced by CT targeted touch in healthy volunteers. This finding holds true for different pain intensities. It is known from animal experiments that C-LMTRs have pain inhibiting capacities [19]. Our results show that this can also be confirmed in humans. Thus, C-LMTRs are not only important for the mediation of pleasantness $[6,10]$ and social aspects of touch [32] but also for the modulation of heat pain under physiological conditions. This assumption is supported by the missing effect of brushing hairy skin in SFN patients with probable C-LTMR degeneration.

The question arises whether the findings can be attributed to $A \beta$-afferent activation. We used a velocity and intensity of brushing that was shown to reliably activate C-LMTRs [6] and to induce a feeling of pleasant touch with a poor spatial localization in two patients with a complete $\mathrm{A} \beta$ fibre loss $[10,33]$. Coding of pleasantness is one of the major functions of CT afferents $[6,10]$. Stroking with a velocity of $3 \mathrm{~cm} / \mathrm{s}$ has also been shown to correlate with the highest pleasantness ratings [33], substantiating a primary activation of CT afferents. Morrison and coworkers investigated patients with hereditary sensory and autonomic neuropathy type V (HSAN V) with a reduction of thin myelinated nerve fibers. The HSAN V patients did not perceive pleasantness of touch by stimulation with a soft brush even though they have 
Table 1 Summary of bold responses for "heat pain only", "heat pain combined with CT targeted touch" and "CT touch", versus control condition showing MNI coordinates, cluster size and peak T-value for healthy subjects (A) and SFN patients (B)

\begin{tabular}{|c|c|c|c|c|}
\hline $\mathrm{H}$ & Brain region & MNI coordinate $(x, y, z)$ & cluster size & peak T-value \\
\hline \multicolumn{5}{|c|}{ A. Bold responses (healthy subjects) } \\
\hline \multicolumn{5}{|c|}{ Heat pain } \\
\hline $\mathrm{R}$ & Insula (ant) & $33,23,1$ & 25 & 10.0226202 \\
\hline R & Middle frontal gyrus, BA 40 & $45,35,25$ & 16 & 9.40863228 \\
\hline $\mathrm{R}$ & Middle frontal gyrus & $33,-1,55$ & 13 & 8.20184994 \\
\hline $\mathrm{R}$ & Supramarginal gyrus, posterior division & $57,-37,43$ & 3 & 7.02497864 \\
\hline $\mathrm{R}$ & Inferior frontal gyrus, pars opercularis & $48,14,13$ & 21 & 8.32530499 \\
\hline R & Angular gyrus; S2* & $45,-49,46$ & 9 & 7.82689619 \\
\hline $\mathrm{R}$ & ACC & $3,26,37$ & 4 & 7.43887186 \\
\hline $\mathrm{R}$ & Paracingulate gyrus & $6,14,46$ & 58 & 8.13667107 \\
\hline L & Paracingulate gyrus & $-6,26,34$ & 8 & 7.75460911 \\
\hline L & Insula (ant) & $-30,23,1$ & 21 & 10.1233358 \\
\hline L & Lateral globus pallidus* & $-18,2,1$ & 4 & 8.00571537 \\
\hline \multicolumn{5}{|c|}{ Heat pain combined with $C T$ targeted touch } \\
\hline $\mathrm{R}$ & Insula (ant.) & $36,26,-5$ & 63 & 8.555808067 \\
\hline $\mathrm{R}$ & ACC & $0,17,49$ & 45 & 7.733303547 \\
\hline $\mathrm{R}$ & Putamen & $24,2,1$ & 8 & 7.900562286 \\
\hline R & Supramarginal gyrus, BA 40; S2* & $57,-40,31$ & 19 & 8.539946556 \\
\hline $\mathrm{R}$ & Middle frontal gyrus, BA 40 & $36,2,49$ & 8 & 7.389503002 \\
\hline $\mathrm{R}$ & Anterior intra-parietal sulcus hIP1 & $42,-49,46$ & 10 & 7.324296474 \\
\hline $\mathrm{R}$ & Inferior frontal gyrus, BA 47 & $42,20,-11$ & 3 & 7.169946671 \\
\hline R & Cerebellum & $30,-58,-32$ & 24 & 7.958759308 \\
\hline L & Insula (ant) & $-30,23,-2$ & 38 & 8.298020363 \\
\hline L & Supramarginal gyrus, BA 40 & $-42,-34,34$ & 5 & 7.888269424 \\
\hline \multicolumn{5}{|c|}{$\mathrm{CT}$ targeted touch } \\
\hline R & Parietal operculum & $48,-34,31$ & 3 & 7.85348225 \\
\hline$L$ & Postcentral gyrus & $-57,-25,34$ & 3 & 6.73631382 \\
\hline
\end{tabular}

\section{B. Bold responses (SFN patients)}

Heat pain

\begin{tabular}{ll}
$R$ & Insula (med.-ant.) \\
$R$ & ACC \\
$R$ & Angular gyrus, BA 40; S2* \\
$R$ & Precuneous cortex \\
$R$ & Lateral occipital cortex \\
$R$ & Lateral occipital cortex \\
$R$ & Broca's area, BA 44 \\
$R$ & Cerebellum, anterior lobe \\
$R$ & Visual cortex, BA 17 \\
$L$ & Insula (ant.) \\
\hline N & Superior parietal lobule \\
$L$ & Anterior intra-parietal sulcus \\
$L$ & Supramarginal gyrus \\
$L$ & Frontal operculum
\end{tabular}

$42,17,-2$
$3,11,46$
$45,-46,49$
$15,-67,43$
$33,-67,40$
$33,-58,46$
$54,11,1$
$27,-55,-20$
$18,-97,4$
$-27,20,-5$
$-30,-52,49$
$-36,-43,37$
$-51,-37,46$
$-42,17,-2$

7.24895525

8.66720772

10.2263985

8.95573997

8.75775337

8.02260399

8.00252247

7.94967175

7.3528986

8.33598518

8.32214737

7.88397264

7.11958122

9.18519974 
Table 1 Summary of bold responses for "heat pain only", "heat pain combined with CT targeted touch" and "CT touch", versus control condition showing MNI coordinates, cluster size and peak T-value for healthy subjects (A) and SFN patients (B) (Continued)

\begin{tabular}{|c|c|c|c|c|}
\hline $\mathrm{H}$ & Brain region & MNI coordinate $(x, y, z)$ & cluster size & peak T-value \\
\hline $\mathrm{L}$ & Cerebellum, posterior lobe & $-27,-62,-29$ & 42 & 10.7323313 \\
\hline$L$ & Cerebellum, posterior lobe & $-6,-76,-23$ & 22 & 8.34745884 \\
\hline L & Inferior cerebellum* & $-24,-61,-50$ & 21 & 10.6803532 \\
\hline \multicolumn{5}{|c|}{ Heat pain combined with $C T$ targeted touch } \\
\hline $\mathrm{R}$ & Supramarginal gyrus, BA 40; S2* & $42,-46,46$ & 168 & 9.750800133 \\
\hline $\mathrm{R}$ & Insula (mid-ant) & $39,17,-2$ & 48 & 9.569821358 \\
\hline $\mathrm{R}$ & Parietal operculum & $60,-28,22$ & 13 & 9.502679825 \\
\hline $\mathrm{R}$ & Precentral gyrus; $\mathrm{S} 1^{*}$ & $48,11,31$ & 14 & 8.683162689 \\
\hline $\mathrm{R}$ & Paracingulate gyrus & $9,14,40$ & 9 & 7.428187847 \\
\hline $\mathrm{R}$ & Cerebellum, anterior lobe (culmen) & $9,-49,-17$ & 3 & 6.953028679 \\
\hline $\mathrm{R}$ & Cerebellum, anterior lobe (culmen) & $30,-49,-26$ & 28 & 8.335325241 \\
\hline $\mathrm{R}$ & Broca's area BA44 & $48,14,16$ & 4 & 7.146142006 \\
\hline $\mathrm{R}$ & Visual cortex, BA 17 & $18,-79,10$ & 4 & 6.931876659 \\
\hline L & Frontal operculum & $-48,17,-2$ & 40 & 8.466537476 \\
\hline $\mathrm{L}$ & Paracingulate gyrus, premotor cortex BA 6 & $0,8,52$ & 11 & 7.569615841 \\
\hline L & Precentral gyrus & $-57,5,4$ & 8 & 9.12651825 \\
\hline L & Superior parietal lobule, anterior intra-parietal sulcus; S2* & $-36,-49,46$ & 3 & 7.223741531 \\
\hline L & Supramarginal gyrus, postcentral gyrus & $-42,-34,4$ & 12 & 8.600948334 \\
\hline$L$ & Cerebellum, posterior lobe & $-33,-64,-44$ & 12 & 8.533157349 \\
\hline L & Cerebellum, posterior lobe (Pyramis) & $-9,-76,-29$ & 15 & 9.127865791 \\
\hline L & Cerebellum posterior lobe (Uvula) & $-21,-67,-26$ & 6 & 7.966376305 \\
\hline L & Cerebellum, anterior lobe & $-36,-55,-29$ & 32 & 8.700092316 \\
\hline \multicolumn{5}{|c|}{$\mathrm{CT}$ targeted touch } \\
\hline $\mathrm{R}$ & Parietal operculum & $48,-31,19$ & 8 & 10.0452766 \\
\hline
\end{tabular}

All activations are shown with $\mathrm{p}$ (peak, FWE-corr) $<0.05$ and cluster size $\geq 3$

*anatomical correspondence in SPM using template

All activations are shown with $p<0.05$ (peak, FWE-correction) and cluster size $\geq 3$. Regions marked with * are named after their anatomical correspondence in SPM using the template avg152T2

intact $A \beta$ afferents [34]. Using this stimulation protocol, we acted on the assumption to mainly observe effects of CT afferents. Moreover, our SFN patients did not show indications of large fibre neuropathy according to the definition of the disease [25]. We performed electroneurography and measured vibration detection thresholds in all patients in order to minimise large fiber involvement. Nevertheless, as we could not directly measure CT fiber activity, we cannot fully exclude possible $A \beta$ fiber involvement in both groups. An example of $A \beta$ fiber mediated heat pain reduction has been reported by Staud et al. with vibro-tactile in healthy individuals and patients with chronic musculoskeletal pain [34].

Very recently published research by Liljencrantz et al. demonstrated that CT targeted touch (using the same stimulation technique as in our study) but not fast brushing ( $\mathrm{A} \beta$ targeted touch) nor vibration stimuli reduced heat pain intensity in healthy volunteers [35], confirming our argumentation above.
Suffering from chronic neuropathic pain central sensitization can be expected in SFN patients that might lead to altered transmission of sensory input. We would expect soft stroking to increase pain intensity, since light sensory stimuli can enter the pain pathway. Since we did not observe a significant modification of heat pain (neither increase nor decrease) by CT stimulation in the patient group we suggest that rather CT fiber loss than alteration of sensory stimuli by central sensitization causes the unaltered pain perception during stroking.

\section{Site of pain modulation}

The cortical activation patterns elicited by pain combined with "CT targeted touch" did not differ between SFN patients and the control group, even though during "CT targeted touch only" we observed significant activations in brain areas processing somatosensory information. Therefore, the major site of C-LTMR pain modulation might not be the brain, but the dorsal horn. 
In the original gate control theory, the spinal cord was considered the site were skin perceptions were modulated before entering the cerebral sensory and affective systems [36]. Many histological studies show a large variety of interneurons and synaptic contacts implying multiple connections of C-LTMRs within the dorsal horn. In mice about $25 \%$ of the neurons in lamina I and II in the dorsal horn, known to be the projection side of C-LTMRs, are inhibitory interneurons [37]. These histological observations are already indicative for a modulation of afferent input in the dorsal horn of the spinal tract. Lu and Perl showed in rats that C-LTMR signaling reduces nociceptive C-fiber signaling at the level of the dorsal horn in Lamina I and II [19]. They reported distinct but overlapping projection areas within the dorsal horn of C-LTMRs, A $\beta$ LTMRs and A $\delta$-LTMRs, further supporting an interaction on the spinal level.

Besides these histo-morphological implications of spinal mechanisms in rodents Mancini et al. presented data with laser evoked potentials in humans, showing a pain relief by touch with von Frey hairs on a segmental level [21]. Recently they added new information, showing, that touch during laser evoked pain changed the laser-evoked potentials (cortical effect) but also the laser evoked blink reflex [38], implying a pain modulation by non-CT targeted touch at the level of the brainstem or below e.g. the spinal cord.

As we did not perform fMRI analysis of the spinal cord, we are not able to report a change of bold responses by $\mathrm{CT}$ stimulation there. A further investigation would be needed to examine a spinal activation pattern by $\mathrm{CT}$ stimulation and heat pain, even though a possible extinction of counteracting effects might occur as well on the spinal level.

\section{Limitations of the study}

We did not find significant differences in fMRI activation between the condition "heat pain" and "heat pain combined with CT targeted touch" in both groups. Besides the implications arguing for a spinal mechanism of CT modulated pain inhibition, another possible explanation could be lack of statistical power, even though a prior power calculation was positive. During the fMRI session, the participants had to evaluate their intensity of pain and perform a motor task (rating). Therefore, the subtle stimulus of $\mathrm{CT}$ activation might be suppressed. But sole CT targeted touch was performed under the same conditions and elicited significant activations of somatosensory brain areas in both groups. We might have achieved a stronger CT stimulation by using a stroking device with skin temperature instead of a brush with neutral temperature [39]. Case and coworkers found different neural processes for coding intensity (posterior insula, primary and secondary somatosensory cortex) and pleasantness of soft touch (pregenual ACC) [12]. These areas are also involved in pain processing [40] which might explain the similarity of the conditions "heat pain" and "heat pain combined with CT targeted touch". Hypothetically, CT targeted touch reduces the heat pain derived activation of the posterior insular and primary sensory cortex by spinal inhibition, but since these areas are not pain specific and activated by sensory stimuli (e.g. CT targeted touch) as well, they might extinct each other (activation and deactivation in the same region) so that there is no resulting difference of bold responses.

The stimulation protocol has not been fully computerized, we relied on an optical trigger to start the stimulation sequences. Therefore, an inaccuracy of synchronism (manual stimulation and start of the MRI acquisition) needs to be taken into account. Because of the preprocessing (e.g. realignment to the mean) we assume no significant interference.

Pain ratings were significantly higher in the SFN patients group. Obviously, SFN patients, which suffer from neuropathic pain have thermal hyperalgesia. To control for a possible effect of pain intensity on CT mediated heat pain modulation we performed an additional experiment (heat pain with and without CT stimulation) on healthy individuals with higher pain intensities, according to those rated by SFN patients $(\mathrm{NRS}=50)$. We also observed a significant reduction of heat pain by CT stimulation which renders a major effect of the different pain intensity on the MRI findings less likely. Nevertheless, high pain intensities in healthy individuals do not control for possible alteration of pain pathways in SFN patients (e.g. central or peripheral sensitization in SFN patients).

To better control against possible $A \beta$ fiber involvement in pain reduction by soft stroking, we should have performed tests with different stroking velocities and pleasantness ratings. But the present protocol included fMRI analysis, hence we had to obey limited investigation times to overcome movement artefacts.

\section{Conclusion}

This study provides new evidence that CT fibers own the ability to reduce heat pain perception in healthy subjects. In a next step, it needs to be investigated if CLTMRs are able to reduce other forms of pain, and how this works. This might open an avenue to new therapy concepts in different pain disorders.

\section{Abbreviations}

CDT: Cold detection threshold; C-LTMR: C-low threshold mechanoreceptive afferents; CNS: Central nervous system; CT: C-tactile, C-touch; fMRI: Functional magnetic resonance imaging; HP: Heat pain; HPT: Heat pain threshold;

IENFD: Intraepidermal nerve fiber density; NRS: Numeric rating scale; 
QST: Quantitative sensory testing; ROI: Region of interest; SFN: Small fiber neuropathy; VAS: Visual analogue scale; WDT: Warm detection threshold

\section{Acknowledgements}

We would like to thank the members of the department for Neuroradiology of the Justus-Liebig-University Giessen and Marion Roß for their technical assistance.

\section{Funding}

This work was supported by the Forschungsförderung $\$ 2$ of the Justus Liebig University, Giessen to HHK.

\section{Availability of data and materials}

The datasets used and/or analysed during the current study are available from the corresponding author on reasonable request.

\section{Authors' contributions}

$\mathrm{HHK}$ and $\mathrm{KH}$ are responsible for the conception and design of the study. $\mathrm{HHK}$, WS, GL and KH were responsible for acquisition of data (e.g. recruitment of patients, electroneurography, quantitative sensory profiles and execution of the experiments). AS was responsible for evaluation of the skin biopsies. BD and ERG supervised the technical settings for fMRI and attended the fMRI experiments. $\mathrm{HHK}, \mathrm{HO}$ and $\mathrm{FB}$ were constantly involved in the analysis and interpretation of data. KH drafted the article. All authors revised the article critically for important intellectual content and approved to the final version of the manuscript.

\section{Ethics approval and consent to participate}

All participants gave their informed written consent according to the latest revision of the Declaration of Helsinki. The study was approved by the local ethics committee of the Justus-Liebig-University, Gießen (105/11).

\section{Consent for publication}

Not applicable.

\section{Competing interests}

The authors declare that they have no competing interests.

\section{Publisher's Note}

Springer Nature remains neutral with regard to jurisdictional claims in published maps and institutional affiliations.

\section{Author details}

'Department of Neurology, Justus Liebig University, Klinikstr. 33, 35392 Giessen, Germany. ${ }^{2}$ Institute of Neuropathology, Justus Liebig University, Arndtstr. 16, 35392 Giessen, Germany. ${ }^{3}$ Department of Radiology, Justus Liebig University, Klinikstr.33, 35392 Giessen, Germany. ${ }^{4}$ Center for Social and Affective Neuroscience, Linköping University, Linköpings Universitet, 58183 Linköping, Sweden. ${ }^{5}$ Department of Neurology, University Medical Center, Johannes Gutenberg-University, Langenbeckstr. 1, 56101 Mainz, Germany. ${ }^{6}$ Department of Neuroradiology, Medical University Innsbruck, Innrain 52, 6020 Innsbruck, Austria.

Received: 6 June 2017 Accepted: 4 September 2017 Published online: 15 September 2017

\section{References}

1. Johansson RS, Trulsson M, Olsson KA, Westberg KG. Mechanoreceptor activity from the human face and oral mucosa. Exp Brain Res. 1988;72(1): 204-8.

2. Vallbo A, Olausson $H$, Wessberg J, Norrsell U. A system of unmyelinated afferents for innocuous mechanoreception in the human skin. Brain Res. 1993;628(1-2):301-4

3. Bessou P, Burgess PR, Perl ER, Taylor CB. Dynamic properties of mechanoreceptors with unmyelinated (C) fibers. J Neurophysiol. 1971;34(1): 116-31.

4. Vallbo AB, Olausson $\mathrm{H}$, Wessberg J. Unmyelinated afferents constitute a second system coding tactile stimuli of the human hairy skin. J Neurophysiol. 1999:81(6):2753-63.

5. Nordin M. Low-threshold mechanoreceptive and nociceptive units with unmyelinated (C) fibres in the human supraorbital nerve. J Physiol. 1990;426:229-40
6. Loken LS, Wessberg J, Morrison I, McGlone F, Olausson H. Coding of pleasant touch by unmyelinated afferents in humans. Nat Neurosci. 2009;12(5):547-8.

7. Li L, Rutlin M, Abraira VE, Cassidy C, Kus L, Gong S, Jankowski MP, Luo W, Heintz N, Koerber HR, et al. The functional organization of cutaneous lowthreshold mechanosensory neurons. Cell. 2011;147(7):1615-27.

8. Craig AD. How do you feel? Interoception: the sense of the physiological condition of the body. Nat Rev Neurosci. 2002;3(8):655-66.

9. Andrew D. Quantitative characterization of low-threshold mechanoreceptor inputs to lamina I spinoparabrachial neurons in the rat. J Physiol. 2010;588(Pt 1):117-24

10. Olausson H, Lamarre $Y$, Backlund $H$, Morin C, Wallin BG, Starck G, Ekholm S, Strigo I, Worsley K, Vallbo AB, et al. Unmyelinated tactile afferents signal touch and project to insular cortex. Nat Neurosci. 2002;5(9):900-4.

11. Liljencrantz J, Bjornsdotter M, Morrison I, Bergstrand S, Ceko M, Seminowicz DA, Cole J, Bushnell MC, Olausson H. Altered C-tactile processing in human dynamic tactile allodynia. Pain. 2013;154(2):227-34.

12. Case LK, Laubacher CM, Olausson H, Wang B, Spagnolo PA, Bushnell MC. Encoding of touch intensity but not pleasantness in human primary somatosensory cortex. J Neurosci. 2016;36(21):5850-60.

13. Sailer U, Triscoli C, Häggblad G, Hamilton P, Olausson H, Croy I. Temporal dynamics of brain activation during $40 \mathrm{~min}$ of pleasant touch. Neurolmage. 2016;139:360-7.

14. Olausson H, Lamarre Y, Backlund H, Morin C, Wallin B, Starck G, Ekholm S, Strigo I, Worsley K, Vallbo $\AA$. Unmyelinated tactile afferents signal touch and project to insular cortex. Nat Neurosci. 2002;5(9):900-4

15. Voos AC, Pelphrey KA, Kaiser MD. Autistic traits are associated with diminished neural response to affective touch. Soc Cogn Affect Neurosci. 2013:8(4):378-86.

16. Bennett RH, Bolling DZ, Anderson LC, Pelphrey KA, Kaiser MD. fNIRS detects temporal lobe response to affective touch. Soc Cogn Affect Neurosci. 2013;9(4):470-6.

17. Gordon I, Voos AC, Bennett RH, Bolling DZ, Pelphrey KA, Kaiser MD. Brain mechanisms for processing affective touch. Hum Brain Mapp. 2013;34(4):914-22.

18. Le Bars $D$. The whole body receptive field of dorsal horn multireceptive neurones. Brain Res Brain Res Rev. 2002:40(1-3):29-44.

19. Lu Y, Perl ER. A specific inhibitory pathway between substantia gelatinosa neurons receiving direct C-fiber input. J Neurosci. 2003;23(25):8752-8.

20. Delfini MC, Mantilleri A, Gaillard S, Hao J, Reynders A, Malapert P, Alonso S, Francois A, Barrere C, Seal R, et al. TAFA4, a chemokine-like protein, modulates injury-induced mechanical and chemical pain hypersensitivity in mice. Cell Rep. 2013;5(2):378-88.

21. Mancini F, Nash T, lannetti GD, Haggard P. Pain relief by touch: a quantitative approach. Pain. 2014;155(3):635-42.

22. Krahe C, Drabek MM, Paloyelis Y, Fotopoulou A. Affective touch and attachment style modulate pain: a laser-evoked potentials study. Philos Trans R Soc Lond Ser B Biol Sci. 2016;371(1708):20160009. doi:10.1098/rstb.2016.0009.

23. Lacomis D. Small-fiber neuropathy. Muscle Nerve. 2002;26(2):173-88.

24. Varney NR, Benton AL. Tactile perception of direction in relation to handedness and familial handedness. Neuropsychologia. 1975;13(4):449-54.

25. Hoeijmakers JG, Faber CG, Lauria G, Merkies IS, Waxman SG. Small-fibre neuropathies-advances in diagnosis, pathophysiology and management. Nat Rev Neurol. 2012;8(7):369-79.

26. Lauria G, Hsieh ST, Johansson O, Kennedy WR, Leger JM, Mellgren SI, Nolano M, Merkies IS, Polydefkis M, Smith AG, et al. European Federation of Neurological Societies/Peripheral Nerve Society Guideline on the use of skin biopsy in the diagnosis of small fiber neuropathy. Report of a joint task force of the European Federation of Neurological Societies and the Peripheral Nerve Society. Eur J Neurol. 2010;17(7):903-12. e944-909

27. Bakkers M, Merkies IS, Lauria G, Devigili G, Penza P, Lombardi R, Herrmans MC, van Nes SI, De Baets M, Faber CG. Intraepidermal nerve fiber density and its application in sarcoidosis. Neurology. 2009;73(14):1142-8.

28. Nagi SS, Rubin TK, Chelvanayagam DK, Macefield VG, Mahns DA. Allodynia mediated by C-tactile afferents in human hairy skin. J Physiol. 2011:589(Pt 16):4065-75.

29. Bakkers M, Merkies IS, Lauria G, Devigili G, Penza P, Lombardi R, Hermans MC, van Nes SI, De Baets M, Faber CG. Intraepidermal nerve fiber density and its application in sarcoidosis. Neurology. 2009;73(14):1142-8.

30. Rolke R, Baron R, Maier C, Tolle TR, Treede RD, Beyer A, Binder A, Birbaumer $\mathrm{N}$, Birklein F, Botefur IC, et al. Quantitative sensory testing in the German 
Research Network on Neuropathic Pain (DFNS): standardized protocol and reference values. Pain. 2006;123(3):231-43.

31. Rolke R, Magerl W, Campbell KA, Schalber C, Caspari S, Birklein F, Treede RD. Quantitative sensory testing: a comprehensive protocol for clinical trials. Eur J Pain. 2006;10(1):77-88.

32. Morrison I, Loken LS, Olausson H. The skin as a social organ. Exp Brain Res. 2010;204(3):305-14.

33. Loken LS, Evert M, Wessberg J. Pleasantness of touch in human glabrous and hairy skin: order effects on affective ratings. Brain Res. 2011;1417:9-15.

34. Staud R, Robinson ME, Goldman CT, Price DD. Attenuation of experimental pain by vibro-tactile stimulation in patients with chronic local or widespread musculoskeletal pain. Eur J Pain. 2011;15(8):836-42.

35. Liljencrantz J, Strigo I, Ellingsen DM, Kramer HH, Lundblad LC, Nagi SS, Leknes S, Olausson H. Slow brushing reduces heat pain in humans. Eur J Pain. 2017;21(7):1173-85

36. Melzack R, Wall PD. Pain mechanisms: a new theory. Science. 1965;150(3699):971-9.

37. Polgar E, Durrieux C, Hughes DI, Todd AJ. A quantitative study of inhibitory interneurons in laminae I-III of the mouse spinal dorsal horn. PLoS One. 2013;8(10):e78309.

38. Mancini F, Beaumont AL, Hu L, Haggard P, lannetti GD. Touch inhibits subcortical and cortical nociceptive responses. Pain. 2015;156(10):1936-44

39. Ackerley R, Backlund Wasling H, Liljencrantz J, Olausson H, Johnson RD, Wessberg J. Human C-tactile afferents are tuned to the temperature of a skin-stroking caress. J Neurosci. 2014;34(8):2879-83.

40. Garcia-Larrea L, Peyron R. Pain matrices and neuropathic pain matrices: a review. Pain. 2013;154(Suppl 1):S29-43.

\section{Submit your next manuscript to BioMed Central and we will help you at every step:}

- We accept pre-submission inquiries

- Our selector tool helps you to find the most relevant journal

- We provide round the clock customer support

- Convenient online submission

- Thorough peer review

- Inclusion in PubMed and all major indexing services

- Maximum visibility for your research

Submit your manuscript at www.biomedcentral.com/submit 